\title{
Ku70 inhibits gemcitabine-induced DNA damage and pancreatic cancer cell apoptosis
}

Jiali Ma ${ }^{1}$, Pingping Hui ${ }^{1}$, Wenying Meng, Na Wang, Shihao Xiang*

Keywords: Pancreatic cancer; Gemcitabine; Ku70; DNA damage; and Chemosensitization.

Abstract. The current study focused on the role of Ku70, a DNA-dependent protein kinase (DNA-PK) complex protein, in pancreatic cancer cell resistance to gemcitabine. In both established cell lines (Mia-PaCa-2 and PANC-1) and primary human pancreatic cancer cells, shRNA/siRNA-mediated knockdown of Ku70 significantly sensitized gemcitabineinduced cell death and proliferation inhibition. Meanwhile, gemcitabine-induced DNA damage and subsequent pancreatic cancer cell apoptosis were also potentiated with Ku70 knockdown. On the other hand, exogenous overexpression of Ku70 in Mia-PaCa-2 cells suppressed gemcitabine-induced DNA damage and subsequent cell apoptosis. In a severe combined immune deficient (SCID) mice Mia-PaCa-2 xenograft model, gemcitabineinduced anti-tumor activity was remarkably pontificated when combined with Ku70 shRNA knockdown in the xenografts. The results of this preclinical study imply that Ku70 might be a primary resistance factor of gemcitabine, and Ku70 silence could significantly chemo- 
sensitize gemcitabine in pancreatic cancer cells.

\section{Introduction}

Pancreatic cancer is still one of the leading causes of cancer-associated mortality in the world [1,2]. It is characterized by rapid disease progression and profound resistance to chemotherapy/radiation $[3,4,5]$. Epidemiological studies demonstrate that the majority of pancreatic cancer patients will succumb within one year after diagnosis [1,2]. Genomic instability is one hallmark of pancreatic cancer, which contributes to chemoresistance [6].

Gemcitabine is the essential adjunct treatment for pancreatic cancer $[4,7,8,9]$. The nucleoside analog is showing clinical activity against pancreatic cancer and other solid tumors [10]. Gemcitabine is catalyzed to the triphosphate within cells, which is then incorporated into DNA to block DNA synthesis [10]. Gemcitabine could also inhibit ribonucleotide reductase, therefore decreasing the deoxynucleotide pools for DNA repair and replication [10]. Gemcitabine's sensitivity directly correlates with the intensity of its incorporation into cellular DNA $[7,10,11,12]$. Thus, gemcitabine treatment would cause significant DNA damage, which is required for subsequent cell apoptosis $[13,14,15,16]$.

DNA-dependent protein kinase (DNA-PK) is primarily composed of three major proteins, including the DNA-PK catalytic subunit (DNA-PKcs), Ku70 and Ku80 [17,18,19]. Although heterodimerization of Ku70 and Ku80 is essential for activation of DNA-PK and DNA repair [20], the two Ku proteins (Ku70 and Ku80) shall have some of the functions that are unique and independent of each other [20]. Ku70 binds directly to the DNA doublestrand break (DSB) ends and participates in non-homologous end-joining (NHEJ) pathway of DSB damages $[17,18,19]$. There are three structural domains for the Ku70 protein, including the $\mathrm{N}$-terminal domain, DNA binding central domain, and C-terminal domain $[13,14,15]$. Recent studies have proposed that Ku70 along with other DNA-PK complex proteins could be important in the chemoresistance of cancer cells $[17,21,22,23]$. The current study focuses on the role of Ku70 in pancreatic cancer cell resistance to gemcitabine. Our results show that Ku70 silence dramatically enhances gemcitabine's sensitivity against human pancreatic cancer cells. 


\section{Materials and methods}

2.1. Reagents. Antibodies of this study were all obtained from Cell Signaling Tech (Shanghai, China). The cell culture reagents were provided by Gibco (Shanghai, China).

2.2. Cell culture. The established human pancreatic cancer cell lines, Mia-PaCa-2 and PANC-1, were purchased from the Cell Bank of Fudan University (Shanghai, China), cells were cultured as previously described [24].

2.3. Primary culture of human pancreatic cancer cells. The surgery-isolated fresh pancreatic cancer specimens from informed-consent patients (no treatment prior to the surgery) were washed and minced. The culture of resolving primary cancer cells was described previously [25]. The protocol was approved by the Institutional Review Board and the Ethics Committee. All studies were conducted in accordance with the principles expressed in the Declaration of Helsinki.

2.4. Western blot assay. Thirty $\mu$ g protein lysates per sample were separated by $10 \%$ SDS-PAGE gel, which were transferred onto PVDF membranes (Bio-Rad, Shanghai, China). The blots, after blocking, were incubated with indicated primary and corresponding second antibodies. The bands were visualized via West Pico ECL reagents (Pierce Biotechnology, Shanghai, China). Protein bands were quantified by the ImageJ software.

\subsection{Quantitative real-time polymerase chain reaction ( $q R T-P C R$ ) assay. Total RNA} was extracted by the SV system (Promega, Shanghai, China), which was then reversetranscribed. cDNA was then amplified by quantitative real-time polymerase chain reaction ("qRT-PCR") assay though the SYBR Green PCR kit (Applied Biosystems, Shanghai, China). Expression of Ku70 mRNA was quantified via the ${ }^{\Delta \Delta} \mathrm{Ct}$ method using $\beta$-actin as the internal control. The primers for Ku70 were 5'-CTGTCCAAGTTGGTCGCTTC-3' (sense) and 5'-CTCTGGAGTTGCCATGATTT-3' (antisense) [26,27]. The primers for Ku80 were 5'C CACCGAGGCACAGTTGAAT- $3^{\prime}$ (sense) and $5^{\prime}$ TCTGTGCAGCAGACACTGAA-3' (antisense) [28]. The primers for $\beta$-actin were 5'TGACGTGGACATCCGCAAAG-3' (sense) and 5 ' CTGGAAGGTGGACAGCGAGG-3' (antisense) [26,27]. PCR was performed in triplicate 
and was conducted using an ABI 7600 System (Applied Biosystems, Shanghai, China). All the primers were synthesized by Genepharm (Shanghai, China).

2.6. MTT assay of cell viability. The cells $\left(5 \times 10^{3}\right)$ were seeded onto 96-well plate. Following applied treatment, cells were incubated with MTT reagent $(0.5 \mathrm{mg} / \mathrm{mL}$, Sigma, Shanghai, China) at $37^{\circ} \mathrm{C}$ for 4 hours, and MTT assay was then performed as described [29]. Optic density (OD) value at $490 \mathrm{~nm}$ was tested as an indicator of cell viability.

2.7. Clonogenic assay of cell proliferation. Following treatment, cells were seeded onto 6-well plates at 1000 cells/well. After 7 days incubation, cells were fixed (with $80 \%$ methanol) and stained (with $0.2 \%$ crystal violet), and colonies were counted manually.

2.8. Y-H2AX FACS assay of cellular DNA damage. After indicated treatment, cells were trypsinized, washed, and fixed in ice-cold ethanol, which were then incubated with a mouse monoclonal anti- $\mathrm{y}-\mathrm{H} 2 \mathrm{AX}$ (Cellular Signaling Tech) antibody overnight, before incubation with a FITC-conjugated anti-mouse secondary antibody (Cell Signaling Tech). Cells were then subjected to FACS assay to determiney-H2AX percentage, which indicates DNA damage intensity [30].

2.9. TUNEL assay of apoptosis. After designated treatment, cell apoptosis was tested by the TUNEL staining assay, the detailed protocol was reported previously [31]. At least 300 cells per treatment in six independent experiments were include to calculate the TUNEL ratio.

2.10. Quantification of apoptosis by ELISA. Following treatment, the cell apoptosis histone DNA ELISA detection kit (Roche, Shanghai, China) was utilized to quantify apoptosis with manufacturer's protocol. ELISA OD value at $450 \mathrm{~nm}$ was recorded as the quantitative indicator of cell apoptosis.

2.11. Stable knockdown of Ku70 and Ku80. A total of seven different Ku70 shRNAs and five different Ku80 shRNAs with non-overlapping sequences were designed by Jikai Gene (Shanghai, China). These shRNAs were packed into the lentiviral GV428 vector. Pancreatic cancer cells were seeded onto 6 -well plates. The lentiviral shRNA $(20 \mu \mathrm{L} / \mathrm{mL}$ 
medium) were added to pancreatic cancer cells for 24 hours. Afterwards, puromycin (5.0 $\mu \mathrm{g} / \mathrm{mL}$, Sigma) was added to select resistant colonies for 2-3 weeks. Expressions of Ku70 and Ku8 in the stable cells were tested by qRT-PCR assay and/or Western blot assay. Of the tested Ku70 shRNAs, three of them efficiently silenced Ku70, which were named Ku70 shRNA (-a/b/c). Meanwhile, two Ku80 shRNAs were effective in downregulating Ku80, and they were named as Ku80 shRNA (-a/-b). The non-sense control lentiviral shRNA (Jikai Gene) was added to the control pancreatic cancer cells.

2.12. siRNA knockdown of Ku70. The two different Ku70 siRNAs were obtained from Ambion ("Ku70 siRNA-a", Austin, TX) and Santa Cruz Biotech ("Ku70 siRNA-b", Shanghai, China). Transfection of Ku70 siRNA was performed by Lipofectamine 2000 (Invitrogen, Shanghai, China) with the distributor's recommendations. The efficiency of siRNA was verified.

2.13 Over-expression of Ku70 and stably cells selection. The full-length human Ku70 cDNA, synthesized via Jikai Gene (Shanghai, China), was cloned into pSuper-flagpuro vector (Addgene, Wuhai, China). The construct or the Empty Vector ("pSuper-Flagpuro") was transfected into Mia-PaCa-2 cells by Lipofectamine 2000 (Invitrogen). Puromycin $(5 \mu \mathrm{g} / \mathrm{mL})$ was again added to select the stable colonies for 5-6 passages. Flagtagged Ku70 was tested by Western blot assay in stable cells.

2.14. Tumor xenograft assay. Female severe combined immune deficient (SCID) mice (4-5 week old and 18-20 g weight) were purchased from Animal Center of Shanghai Biological Science Institution (Shanghai, China). Ten million Mia-PaCa-2 cells, stably expressing indicated shRNA, were subcutaneously injected into the right flank of each mouse. Palpable tumors started to appear in 3-5 weeks. Following the treatment, the size of the tumors was measured by caliper every week, and tumor volume was calculated using the following formula: $\pi / 6 \times$ width $2 \times$ length [32]. Mice body weights were also recorded every week. All animal procedures were performed according to the IACUC guidelines upon approval by all authors' Institutional Review Board.

2.15. Statistical analysis. The data presented were means \pm standard deviation (SD). Statistical differences were analyzed by one-way ANOVA followed by multiple comparisons 
performed with post hoc Bonferroni test (SPSS).

\section{Results}

\subsection{Ku70 knockdown sensitizes gemcitabine-induced killing of pancreatic cancer cells}

We initially designed a total of seven non-overlapping Ku70 shRNAs. Three of them were effective. These shRNAs were labeled as: sh-Ku70 (a), sh-Ku70 (b) and sh-Ku70 (c) (See methods). qRT-PCR assay results and Western blot assay results in Figure 1A demonstrated that the three shRNAs indeed dramatically downregulated Ku70 protein and mRNA expression in Mia-PaCa-2 cells. Expression of Ku80 was not altered by the Ku70 shRNAs (Figure 1A). Remarkably, Ku70 knockdown dramatically sensitized gemcitabineinduced cytotoxicity in Mia-PaCa-2 cells, causing profound cell death (MTT OD reduction, Figure 1B) and proliferation inhibition (colony formation suppression, Figure 1C). Intriguingly, shRNA knockdown of Ku70 by itself failed to affect Mia-PaCa-2 cell survival and proliferation (Figure 1B and C).

Similar experiments were also performed in the other established human pancreatic cancer cell line: PANC-1. Again, the three shRNAs efficiently knocked down Ku70 (but not Ku80) in PANC-1 cells (Figure 1D). Gemcitabine-induced killing of PANC-1 cells, tested by MTT OD reduction, was again significantly potentiated when Ku70 was silenced (Figure 1E). In the primary pancreatic cancer cells, siRNA strategy was applied to knockdown Ku70. Results displayed that the two applied siRNAs decreased Ku70 protein and mRNA expressions in the primary cancer cells (Figure 1F). Consequently, gemcitabine-induced cancer cell death (tested by viability reduction) was also significantly augmented (Figure 1G). These results suggest that Ku70 silence sensitizes gemcitabine-induced killing of pancreatic cancer cells.

\subsection{Ku70 knockdown facilitates gemcitabine-induced DNA damage and pancreatic cancer cell apoptosis}

Gemcitabine induces DNA damage to cancer cells, which leads to subsequent cell 
apoptosis [30,33,34,35]. Ku70 could repair DNA damage under a number of stimuli $[13,14,15,16]$. We therefore tested the level of DNA damage in Ku70-silenced cells. Results showed that treatment with gemcitabine in Mia-PaCa2 cells induced significant DNA damage, which was evidenced by an increased of $\mathrm{y}-\mathrm{H} 2 \mathrm{AX}$ staining [16,30] (Figure 2A). Remarkably, shRNA-mediated knockdown of Ku70 dramatically sensitized gemcitabineinduced DNA damage, causing a profound increase number of cells with $\mathrm{y}-\mathrm{H} 2 \mathrm{AX}$ staining (Figure 2A).

Next, we tested gemcitabine-induced apoptosis in Ku70-silenced pancreatic cancer cells. Two independent assays were performed, including histone-DNA ELISA assay and TUNEL staining assay. Results from both assays showed that gemcitabine alone only induced minor apoptosis activation in Mia-PaCa2 cells (Figure 2B and C). Remarkably, Ku70 shRNA knockdown significantly potentiated gemcitabine-induced apoptosis activation (Figure 2B and C). All three applied Ku70 shRNAs achieved similar results (Figure 2A-C). The similar experiments were also performed in PANC-1 cells, and Ku70 knockdown again dramatically sensitized gemcitabine-induced apoptosis activation (Figure 2D). In the primary pancreatic cancer cells, Ku70 siRNA (-a/-b) similarly facilitated gemcitabineinduced DNA damage (Figure 2E). Meanwhile, a profound apoptosis by gemcitabine was only observed in Ku70 knockdown cells (Figure 2F). Together, these results suggest that Ku70 knockdown facilitates gemcitabine-induced DNA damage and apoptosis in pancreatic cancer cells. Significantly, we found that knockdown of Ku80, by two distant Ku80 shRNAs ("-a/-b") (Supplementary Figure 1A), also potentiated gemcitabine-induced viability reduction (MTT assay, Supplementary Figure 1B) and apoptosis (Histone DNA ELISA assay, Supplementary Figure 1C) in MIA-PaCa-2 cells. However, Ku70 knockdown was significantly more efficient than Ku80 knockdown in facilitating gemcitabine-induced killing of MIA-PaCa-2 cells (Supplementary Figure 1B and $\mathbf{C})$.

\subsection{Forced overexpression of Ku70 deceases gemcitabine's sensitivity in pancreatic cancer cells}

Based on the above results, we would speculate that Ku70 overexpression should decrease gemcitabine's sensitivity. As described, the Ku70 expression vector (see method) was introduced to the MiaPaCa-2 cells. Through puromycin selection, two stable MiaPaCa-2 cell lines expressing the construct were established, named "wt-Ku70-a" and 
"wt-Ku70-b". Western blot assay and RT-PCR assay results in Figure 3A confirmed Ku70 protein (flag-tagged) and mRNA overexpression in the stable cells. Significantly, as demonstrated in Figure 3B, gemcitabine-induced DNA damage, or $\gamma-\mathrm{H} 2 \mathrm{AX}$ staining, was decreased sharply in Ku70-overexpressed cells. Consequently, gemcitabine-induced MiaPaCa-2 cell viability reduction (Figure 3C), colony inhibition (Figure 3D) and apoptosis (Figure 3E) were all attenuated with Ku70 overexpression. Thus, exogenous Ku70 expression could further decrease gemcitabine's sensitivity in pancreatic cancer cells.

\subsection{Ku70 knockdown sensitizes gemcitabine-induced anti-tumor activity in vivo}

At last, we applied the SCID mice Mia-PaCa-2 xenograft mode to test Ku70's effect on gemcitabine-induced anti-tumor activity in vivo. Same amount (10 million cells per mouse) of stable Mia-PaCa-2 cells, expressing non-sense control shRNA or the Ku70 shRNA (-a/b), were injected to the SCID mice. Within three weeks, Mia-PaCa-2 xenografts were established with the volume around $100 \mathrm{~mm}^{3}$. As demonstrated in Figure 4A, intraperitoneal injection of gemcitabine (50 mg/kg body weight, daily) only moderately inhibited growth of Mia-PaCa-2 xenografts with control shRNA. Remarkably, gemcitabine induced a much potent growth inhibition of Mia-PaCa-2 xenografts expressing Ku70 shRNA (-a/-b, Figure 4A). Results in Figure 4B confirmed that, with same gemcitabine administration, daily growth of Ku70-silenced tumors was dramatically slower than that of control shRNA tumors. Notably, Ku70 shRNA alone didn't affect Mia-PaCa-2 xenograft growth in the SCID mice (Data not shown).

Intriguingly, the mice body weight was not significantly different between the groups (Figure 4C), indicating that these regimens were safe to the SCID mice. We also failed to detect any significant or apparent toxicity (Data not shown). We tested Ku70 protein expression in the tumor lysates. Results showed that at day-7 and day-14 after initial gemcitabine administration, Ku70 protein was indeed dramatically downregulated in Ku70 shRNA-expressing tumors (Figure 4D). Once again, Ku70 shRNA didn't affect Ku80 expression (Figure 4D). Together, these results indicate that Ku70 knockdown sensitizes gemcitabine-induced anti-tumor activity in vivo.

\section{Discussions}


The nucleoside analog gemcitabine suppresses DNA replication through replacing deoxycytidine's 2 ' carbon to fluorine atoms $[9,11]$. Its cytotoxicity against human pancreatic cancer cells is limited due to development of chemoresistance $[9,11]$. Gemcitabine-induced cell apoptosis is associated with DNA damage in the cancer cells [30,34]. Inhibition or repair of gemcitabine-induced DNA damage could significantly decrease cancer cell apoptosis $[30,34]$. In this study, we propose that Ku70 may be involved in repairing DNA damage by gemcitabine, and protects cells from apoptosis.

Recent studies have proposed an oncogenic role of Ku70 in cancer cell progression $[14,16,26,36]$. In the current study, we propose that Ku70 could also be important for gemcitabine's resistance in human pancreatic cancer cells. Knockdown of Ku70, by targeted shRNA or siRNA, significantly sensitized gemcitabine-induced DNA damage and subsequent pancreatic cancer cell death/apoptosis. On the other hand, forced overexpression of Ku70 decreased gemcitabine-induced DNA damage, and pancreatic cancer cell. In vivo, Ku70 shRNA significantly sensitized gemcitabine-induced tumor growth inhibition in SCID mice. These results suggest that Ku70 could be a key gemcitabine resistance factor and a promising oncotarget of pancreatic cancer.

In the current study, although Ku80 knockdown also potentiated gemcitabine-induced killing of MIA-PaCa-2 cells. However, gemcitabine-sensitization by knockdown of Ku80 was less effective than knockdown of Ku70. These results imply that DNA repair shall only be one of many mechanisms responsible for Ku70-mediated actions on gemcitabine. Other Ku80-/DNA repair-independent mechanisms should also be involved. As a matter of fact, besides DNA repair, existing evidences have suggested that Ku70 protein is also important in regulating a number of other key cellular functions, including telomere maintenance, gene transcription and apoptosis [20]. For example, it is known that Ku70 could interact with and inhibit Bax, thus sequestering mitochondrial apoptosis pathway $[37,38]$. Such effect by Ku70 is intriguingly Ku80-independent [37,38]. In line with our findings, other groups have already proposed a role of Ku70 in chemoresistance [16,39]. For instance, siRNA-mediated knockdown of Ku70 significantly enhanced human cancer cell sensitivity to topoisomerase II inhibitor [16]. Rashmi et al., demonstrated that Ku70 is important for curcumin's resistance [39]. Future studies will be needed to explore the mechanisms of Ku70-mediated 
gemcitabine-resistance in pancreatic cancer cells, and the involvement of DNA repairindependent actions.

In summary, the results of this preclinical study demonstrated the efficacy of Ku70 silence in sensitizing pancreatic cancer cells to gemcitabine. These results are not unexpected from a mechanistic standpoint, and should have important translational value for the pancreatic cancer treatment.

Acknowledgments. This work is supported by the National Science Foundation of China. Conflict of interests. The authors declare that they have no conflict of interests.

\section{References}

[1] R.L. Siegel, K.D. Miller, A. Jemal, Cancer statistics, 2015, CA Cancer J Clin 65 (2015) 5-29.

[2] R. Siegel, J. Ma, Z. Zou, A. Jemal, Cancer statistics, 2014, CA Cancer J Clin 64 (2014) 9-29.

[3] A. Seicean, L. Petrusel, R. Seicean, New targeted therapies in pancreatic cancer, World J Gastroenterol 21 (2015) 6127-6145.

[4] A. Akinleye, C. Iragavarapu, M. Furqan, S. Cang, D. Liu, Novel agents for advanced pancreatic cancer, Oncotarget 6 (2015) 39521-39537.

[5] M. Hidalgo, Pancreatic cancer, N Engl J Med 362 (2010) 1605-1617.

[6] E. Costello, J.P. Neoptolemos, Pancreatic cancer in 2010: new insights for early intervention and detection, Nat Rev Gastroenterol Hepatol 8 (2011) 71-73.

[7] M. Loos, J. Kleeff, H. Friess, M.W. Buchler, Surgical treatment of pancreatic cancer, Ann N Y Acad Sci 1138 (2008) 169-180.

[8] M. Ducreux, V. Boige, D. Malka, Treatment of advanced pancreatic cancer, Semin Oncol 34 (2007) S25-30.

[9] F. Eckel, G. Schneider, R.M. Schmid, Pancreatic cancer: a review of recent advances, Expert Opin Investig Drugs 15 (2006) 1395-1410.

[10] D.S. Gesto, N.M. Cerqueira, P.A. Fernandes, M.J. Ramos, Gemcitabine: a critical nucleoside for cancer therapy, Curr Med Chem 19 (2012) 1076-1087.

[11] D.D. Von Hoff, T. Ervin, F.P. Arena, E.G. Chiorean, J. Infante, M. Moore, T. Seay, S.A. Tjulandin, W.W. Ma, M.N. Saleh, M. Harris, M. Reni, S. Dowden, D. Laheru, N. Bahary, R.K. Ramanathan, J. Tabernero, M. Hidalgo, D. Goldstein, E. Van Cutsem, X. Wei, J. Iglesias, M.F. Renschler, Increased survival in pancreatic cancer with nab-paclitaxel plus gemcitabine, N Engl J Med 369 (2013) 1691-1703.

[12] W. Plunkett, P. Huang, Y.Z. Xu, V. Heinemann, R. Grunewald, V. Gandhi, Gemcitabine: metabolism, mechanisms of action, and self-potentiation, Semin Oncol 22 (1995) 3-10.

[13] S.E. Lee, J.K. Moore, A. Holmes, K. Umezu, R.D. Kolodner, J.E. Haber, Saccharomyces Ku70, mre11/ rad50 and RPA proteins regulate adaptation to G2/M arrest after DNA damage, Cell 94 (1998) 399-409.

[14] S. Jin, D.T. Weaver, Double-strand break repair by Ku70 requires heterodimerization with Ku80 and DNA binding functions, EMBO J 16 (1997) 6874-6885.

[15] P. Bundock, H. van Attikum, P. Hooykaas, Increased telomere length and hypersensitivity to DNA damaging agents in an Arabidopsis KU70 mutant, Nucleic Acids Res 30 (2002) 3395-3400.

[16] I.S. Ayene, L.P. Ford, C.J. Koch, Ku protein targeting by Ku70 small interfering RNA enhances human cancer cell response to topoisomerase II inhibitor and gamma radiation, Mol Cancer Ther 4 (2005) 529-536.

[17] D.A. Dungl, E.N. Maginn, E.A. Stronach, Preventing Damage Limitation: Targeting DNA-PKcs and DNA Double-Strand Break Repair Pathways for Ovarian Cancer Therapy, Front Oncol 5 (2015) 240. 
[18] P. Bouchaert, S. Guerif, C. Debiais, J. Irani, G. Fromont, DNA-PKcs expression predicts response to radiotherapy in prostate cancer, Int J Radiat Oncol Biol Phys 84 (2012) 1179-1185.

[19] T.A. Dobbs, J.A. Tainer, S.P. Lees-Miller, A structural model for regulation of NHEJ by DNA-PKcs autophosphorylation, DNA Repair (Amst) 9 (2010) 1307-1314.

[20] C. Gullo, M. Au, G. Feng, G. Teoh, The biology of Ku and its potential oncogenic role in cancer, Biochim Biophys Acta 1765 (2006) 223-234.

[21] C.H. Kim, S.J. Park, S.H. Lee, A targeted inhibition of DNA-dependent protein kinase sensitizes breast cancer cells following ionizing radiation, J Pharmacol Exp Ther 303 (2002) 753-759.

[22] H. Hu, Y. Gu, Y. Qian, B. Hu, C. Zhu, G. Wang, J. Li, DNA-PKcs is important for Akt activation and gemcitabine resistance in PANC-1 pancreatic cancer cells, Biochem Biophys Res Commun 452 (2014) 106-111.

[23] D. Davidson, L. Amrein, L. Panasci, R. Aloyz, Small Molecules, Inhibitors of DNA-PK, Targeting DNA Repair, and Beyond, Front Pharmacol 4 (2013) 5.

[24] Y. Jiang, Q. Meng, B. Chen, H. Shen, B. Yan, B. Sun, The small-molecule IAP antagonist AT406 inhibits pancreatic cancer cells in vitro and in vivo, Biochem Biophys Res Commun 478 (2016) 293-299.

[25] S. Minjie, H. Defei, H. Zhimin, W. Weiding, Z. Yuhua, Targeting pancreatic cancer cells by a novel hydroxamate-based histone deacetylase (HDAC) inhibitor ST-3595, Tumour Biol 36 (2015) 9015-9022.

[26] Y. Lu, J. Gao, Down-expression pattern of Ku70 and p53 coexisted in colorectal cancer, Med Oncol 32 (2015) 98.

[27] Y. Lu, J. Gao, Downregulated Ku70 and ATM associated to poor prognosis in colorectal cancer among Chinese patients, Onco Targets Ther 7 (2014) 1955-1961.

[28] D. Qi, Y. Hu, J. Li, T. Peng, J. Su, Y. He, W. Ji, Hyperthermia Induces Apoptosis of 786-O Cells through Suppressing Ku80 Expression, PLoS One 10 (2015) e0122977.

[29] C. Cao, X. Huang, Y. Han, Y. Wan, L. Birnbaumer, G.S. Feng, J. Marshall, M. Jiang, W.M. Chu, Galpha(i1) and Galpha(i3) are required for epidermal growth factor-mediated activation of the Akt-mTORC1 pathway, Sci Signal 2 (2009) ra17.

[30] B. Ewald, D. Sampath, W. Plunkett, H2AX phosphorylation marks gemcitabine-induced stalled replication forks and their collapse upon S-phase checkpoint abrogation, Mol Cancer Ther 6 (2007) 1239-1248.

[31] C. Zhou, Z. Chen, X. Lu, H. Wu, Q. Yang, D. Xu, Icaritin activates JNK-dependent mPTP necrosis pathway in colorectal cancer cells, Tumour Biol 37 (2016) 3135-3144.

[32] L.S. Qin, P.F. Jia, Z.Q. Zhang, S.M. Zhang, ROS-p53-cyclophilin-D signaling mediates salinomycininduced glioma cell necrosis, J Exp Clin Cancer Res 34 (2015) 57.

[33] L.A. Parsels, M.A. Morgan, D.M. Tanska, J.D. Parsels, B.D. Palmer, R.J. Booth, W.A. Denny, C.E. Canman, A.J. Kraker, T.S. Lawrence, J. Maybaum, Gemcitabine sensitization by checkpoint kinase 1 inhibition correlates with inhibition of a Rad51 DNA damage response in pancreatic cancer cells, Mol Cancer Ther 8 (2009) 45-54.

[34] G. Achanta, H. Pelicano, L. Feng, W. Plunkett, P. Huang, Interaction of p53 and DNA-PK in response to nucleoside analogues: potential role as a sensor complex for DNA damage, Cancer Res 61 (2001) 8723-8729.

[35] M. Crul, R.C. van Waardenburg, S. Bocxe, M.A. van Eijndhoven, D. Pluim, J.H. Beijnen, J.H. Schellens, DNA repair mechanisms involved in gemcitabine cytotoxicity and in the interaction between gemcitabine and cisplatin, Biochem Pharmacol 65 (2003) 275-282.

[36] J.W. Lim, H. Kim, K.H. Kim, Expression of Ku70 and Ku80 mediated by NF-kappa B and cyclooxygenase- 2 is related to proliferation of human gastric cancer cells, J Biol Chem 277 (2002) 46093-46100.

[37] H.Y. Cohen, S. Lavu, K.J. Bitterman, B. Hekking, T.A. Imahiyerobo, C. Miller, R. Frye, H. Ploegh, B.M. Kessler, D.A. Sinclair, Acetylation of the C terminus of Ku70 by CBP and PCAF controls Bax-mediated apoptosis, Mol Cell 13 (2004) 627-638.

[38] M. Sawada, W. Sun, P. Hayes, K. Leskov, D.A. Boothman, S. Matsuyama, Ku70 suppresses the apoptotic translocation of Bax to mitochondria, Nat Cell Biol 5 (2003) 320-329.

[39] R. Rashmi, S. Kumar, D. Karunagaran, Ectopic expression of Bcl-XL or Ku70 protects human colon cancer cells (SW480) against curcumin-induced apoptosis while their down-regulation potentiates it, Carcinogenesis 25 (2004) 1867-1877.

\section{Figure legends}

\section{Figure 1. Ku70 knockdown sensitizes gemcitabine-induced killing of pancreatic} cancer cells. Puromycin-selected stable MIA-PaCa-2 cells or PANC-1 cells, expressing 
Ku70 shRNA ["sh-Ku70(a/b/c)"] or non-sense control shRNA ("sh-NC"), as well as the parental cells ("Parental"), were treated with/out designated concentration of gemcitabine, Ku70 protein and mRNA expressions were tested Western blot assay and qRT-PCR assay, respectively (A and D); Cell viability (MTT assay, B and E) and colony formation (C) were also examined. Primary pancreatic cancer cells, transfected with Ku70 siRNA ["si-Ku70(a/ b)"] or non-sense control siRNA ("si-NC"), were treated with/out applied gemcitabine, Ku70 expression was shown (F); Cell viability was also tested $(\mathbf{G})$. Experiments in this figure were repeated four times, with similar results obtained. Bars stand for mean \pm SD (Same for all figures). Relative Ku70/Ku80 protein expression was normalized to Tubulin (A, D and F). * $\boldsymbol{p}<0.05$ vs. untreated control group ("Ctrl"). ${ }^{\#} \boldsymbol{p}<0.05$ vs. "sh-NC" or "si-NC" group.

Figure 2. Ku70 knockdown facilitates gemcitabine-induced DNA damage and pancreatic cancer cell apoptosis. Puromycin-selected stable MIA-PaCa-2/PANC-1 cells, expressing Ku70 shRNA ["sh-Ku70(a/b/c)"] or non-sense control shRNA ("sh-NC"), were treated with/out designated gemcitabine, DNA damage was tested by $\mathrm{Y}-\mathrm{H} 2 \mathrm{AX}$ FACS assay (A); Cell apoptosis was tested by the listed assays (B-D). Primary pancreatic cancer cells, with Ku70 siRNA ["si-Ku70(a/b)"] or non-sense control siRNA ("si-NC"), were treated with/ out gemcitabine (100 nM), DNA damage (E) and cell apoptosis (F) were tested. Experiments in this figure were repeated three times, with similar results obtained. ${ }^{*} \boldsymbol{p}<$ 0.05 vs. untreated control group ("Ctrl"). ${ }^{\#} \boldsymbol{p}<0.05$ vs. "sh-NC" or "si-NC" group.

Figure 3. Forced overexpression of Ku70 deceases gemcitabine's sensitivity in pancreatic cancer cells. Puromycin-selected stable MIA-PaCa-2 cells, expression Ku70 vector [wt-Ku70 (-a/-b), two lines] or the Empty Vector ("pSuper-flag-puro"), were treated with/out gemcitabine for designated time, Ku70 expression (A), DNA damage (B), cell viability (C), colony formation (D) and cell apoptosis (E) were tested by the listed assays. Relative Ku70/Ku80 protein expression was normalized to Tubulin (A). Experiments in this figure were repeated three times, with similar results obtained. ${ }^{*} \boldsymbol{p}<0.05$ vs. untreated control group (“Ctrl”). ${ }^{\#} \boldsymbol{p}<0.05$ vs. "Empty vector" group.

Figure 4. Ku70 knockdown sensitizes gemcitabine-induced anti-tumor activity in vivo. SCID mice ( $n=10$ per group), bearing MIA-PaCa-2 tumors expressing Ku70 shRNA ["sh-Ku70(a/b)"] or non-sense control shRNA ("sh-NC"), were treated with vehicle control 
("Saline") or i.p. administration of gemcitabine ("Gem", $50 \mathrm{mg} / \mathrm{kg}$ body weight, daily, for 28 days), tumor volumes (A) and mice body weights (C) were measured weekly; Estimated daily tumor growth was also calculated (B). Day-7 and day-14 after initial gemcitabine administration, one tumor per group was isolated, expressions of listed proteins in tumor lysates were tested (D). Relative Ku70/Ku80 protein expression was normalized to Erk1/2 (D). * $\boldsymbol{p}<0.05$ vs. "sh-NC+Saline" group. ${ }^{\#} \boldsymbol{p}<0.05$ vs. "sh-NC+Gem" group. 
Figure 1.

A

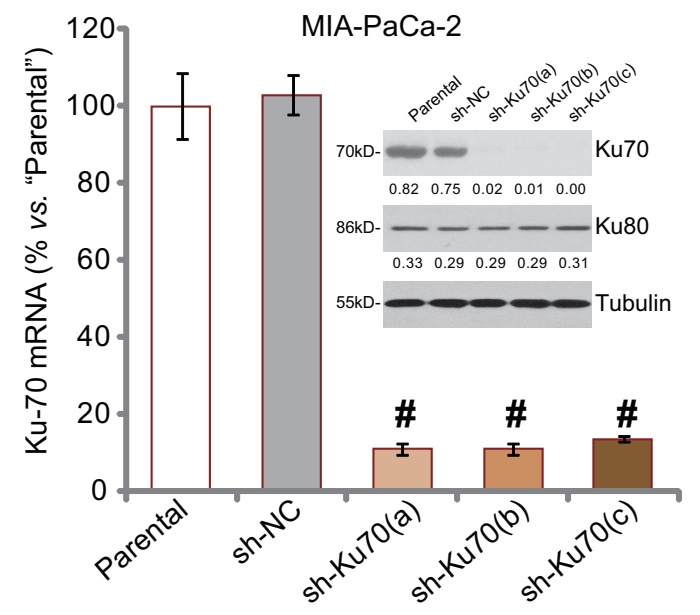

D

PANC-1
E

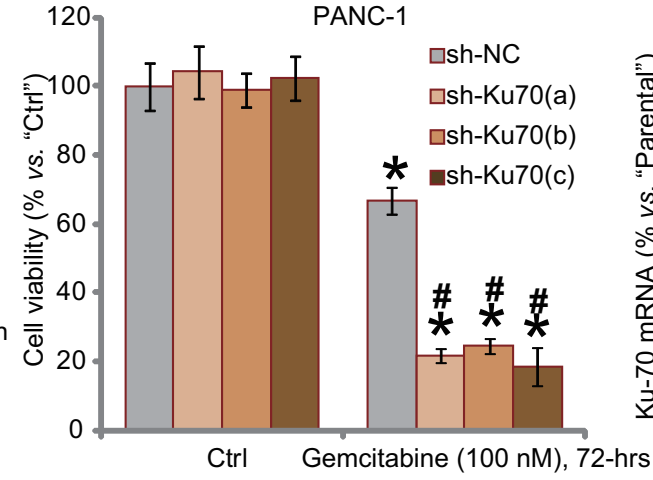

F
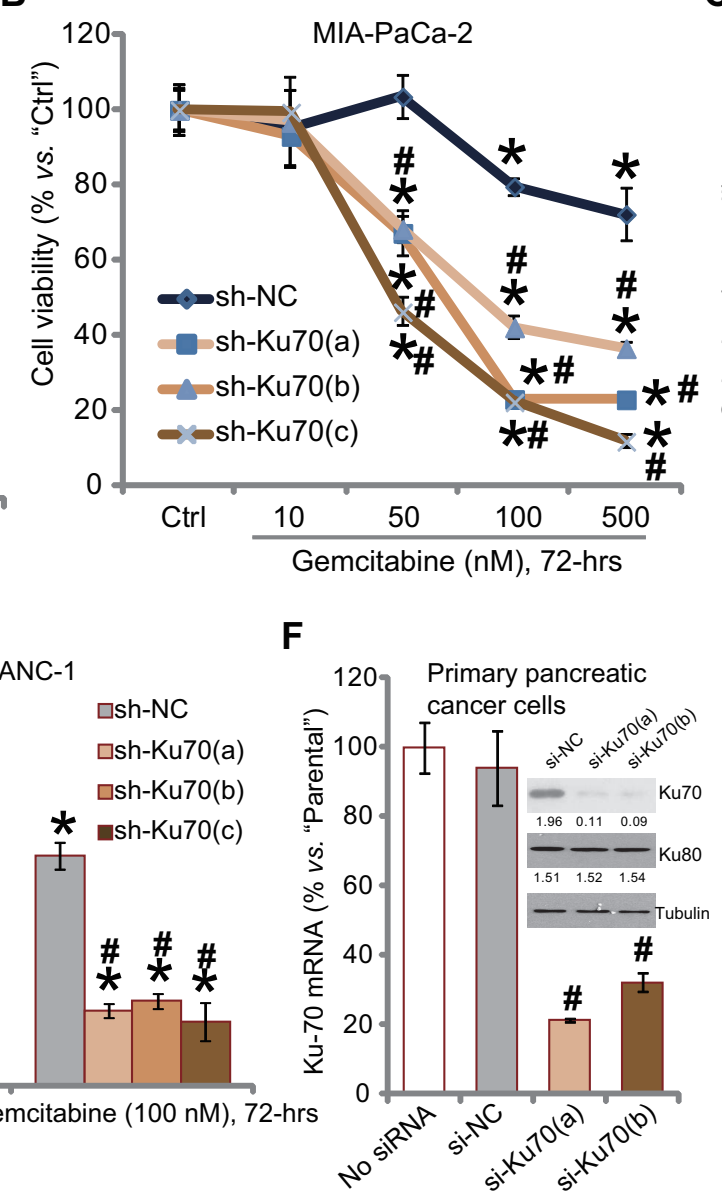

C

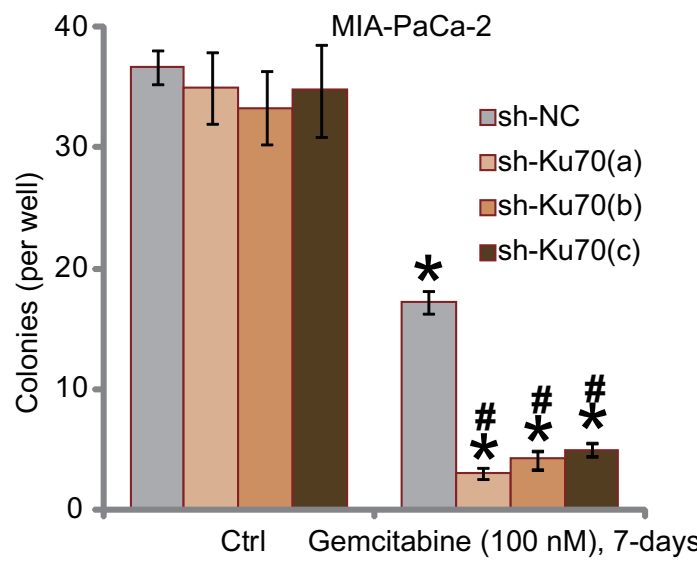

G

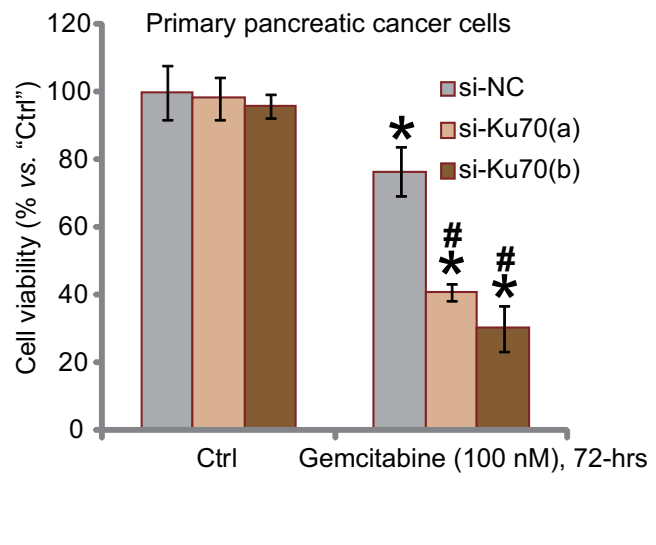


Figure 2.

A

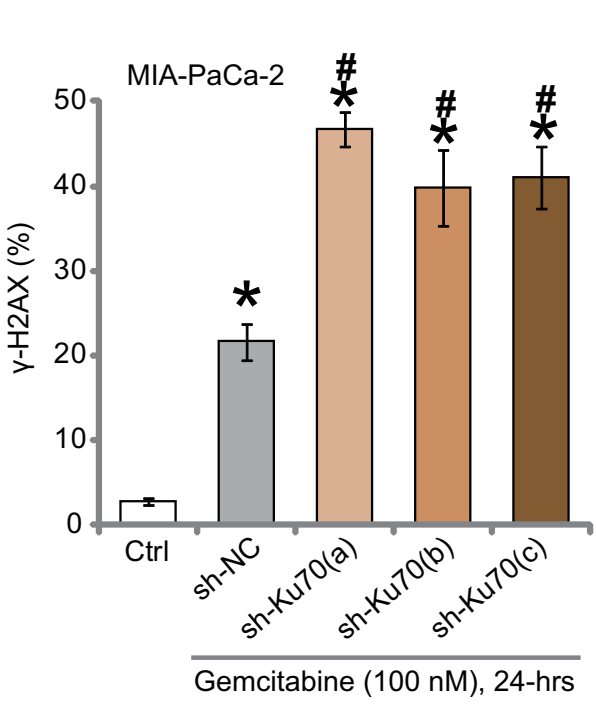

B C

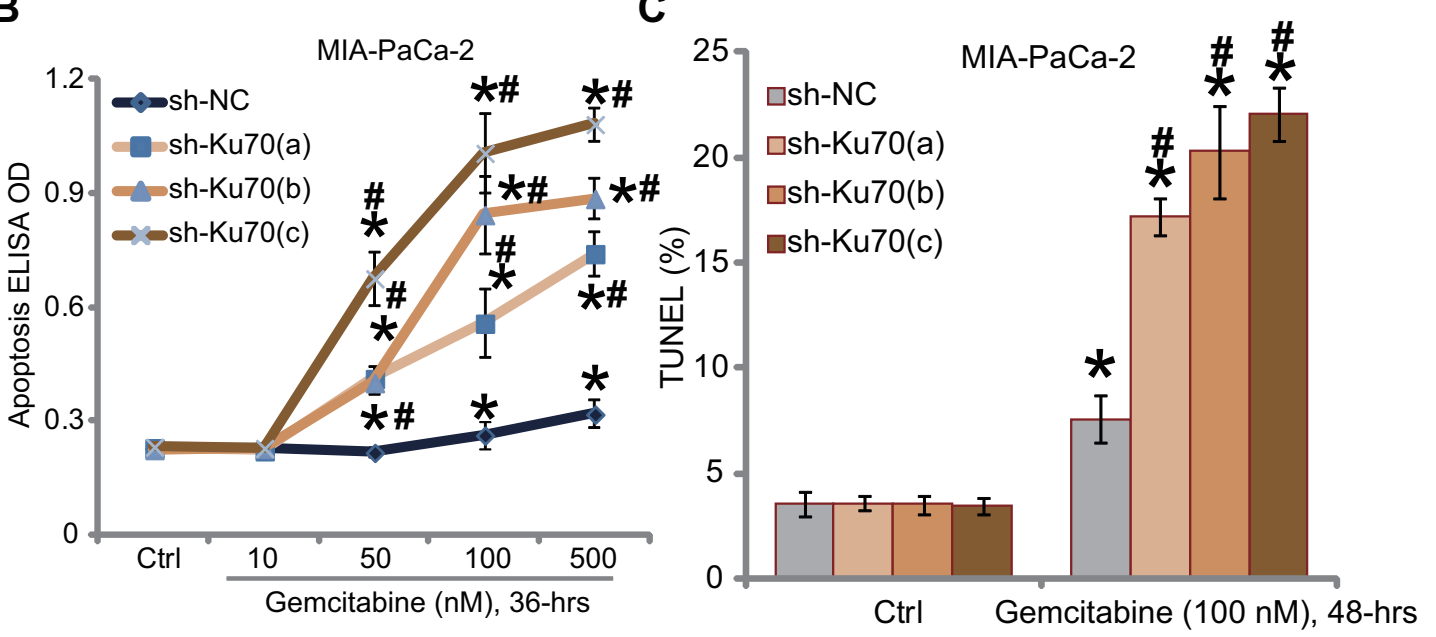

E

$F$

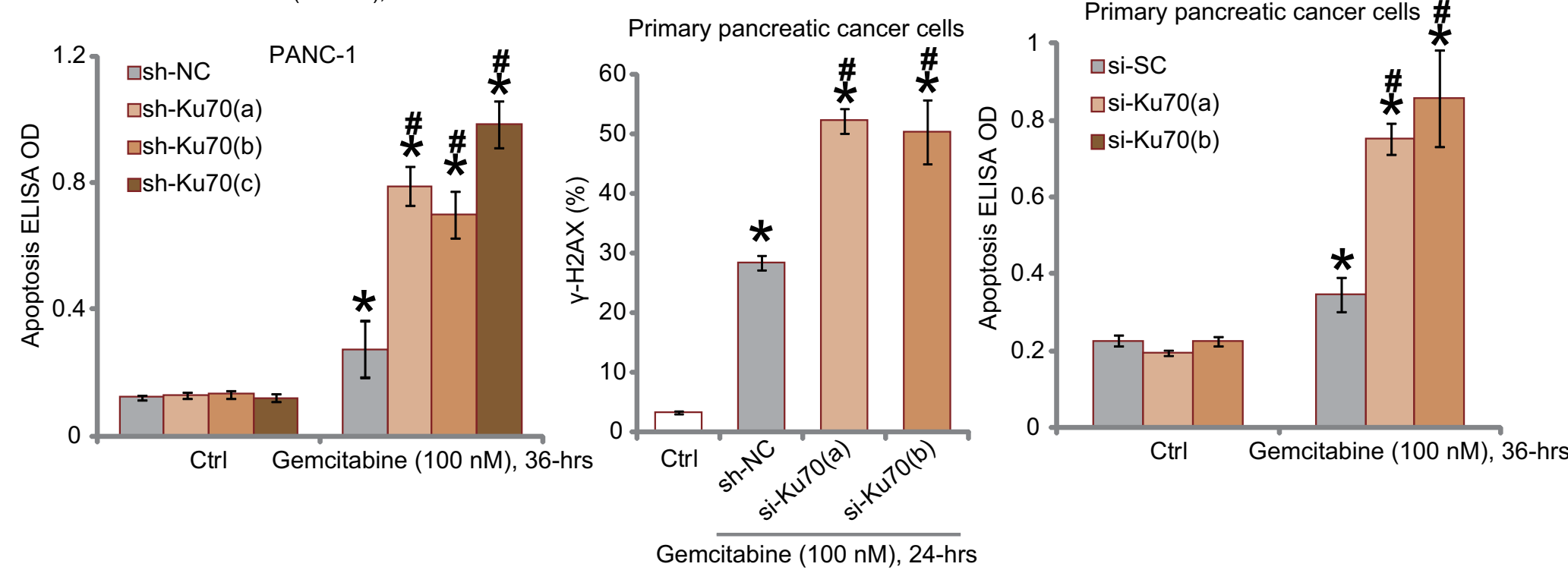


Figure 3.

A

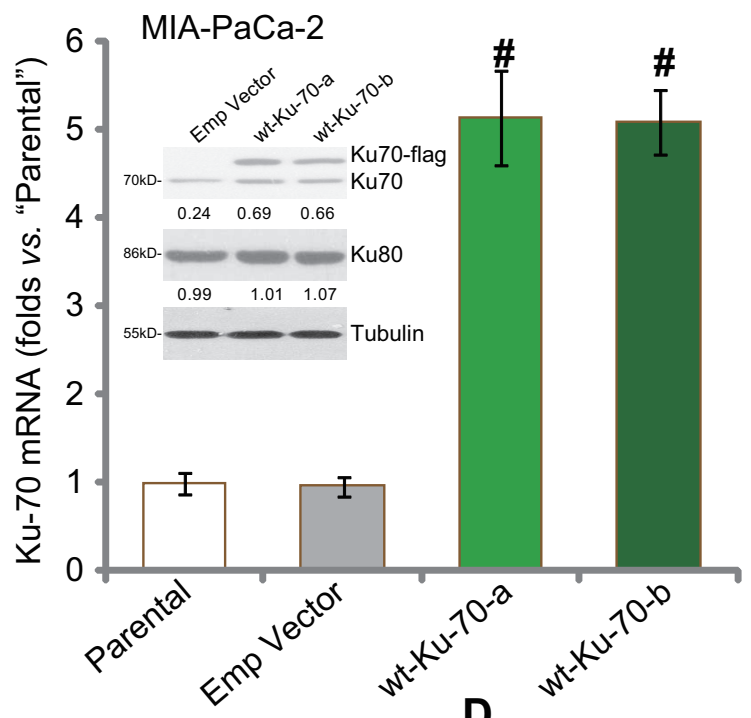

C
B

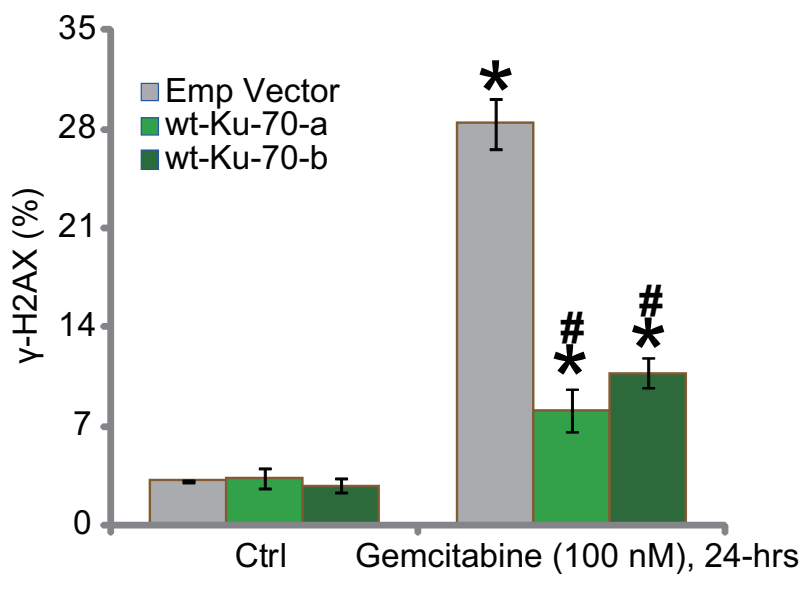

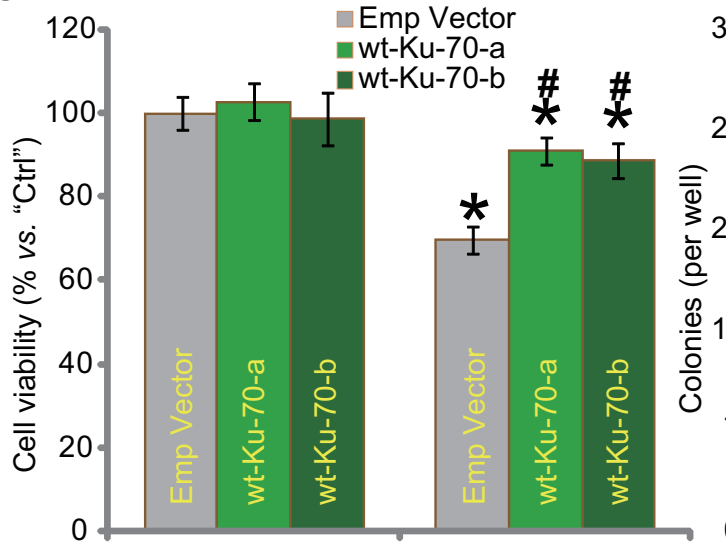

Ctrl Gemcitabine $(100 \mathrm{nM})$, 72-hrs

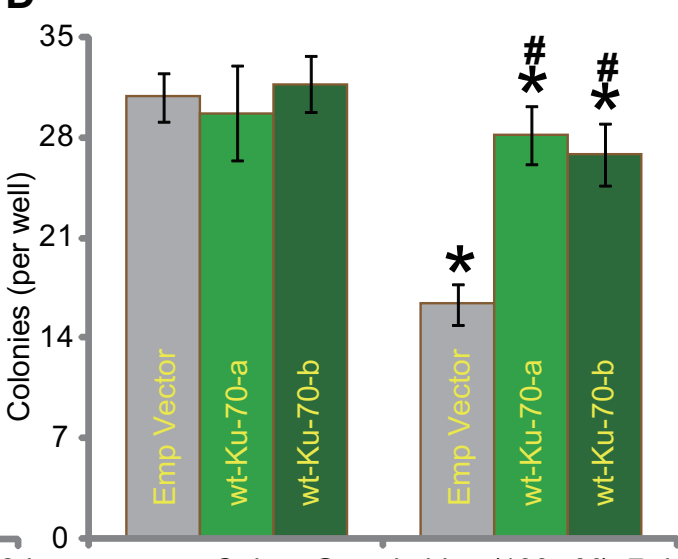

Ctrl Gemcitabine (100 nM), 7-days
E

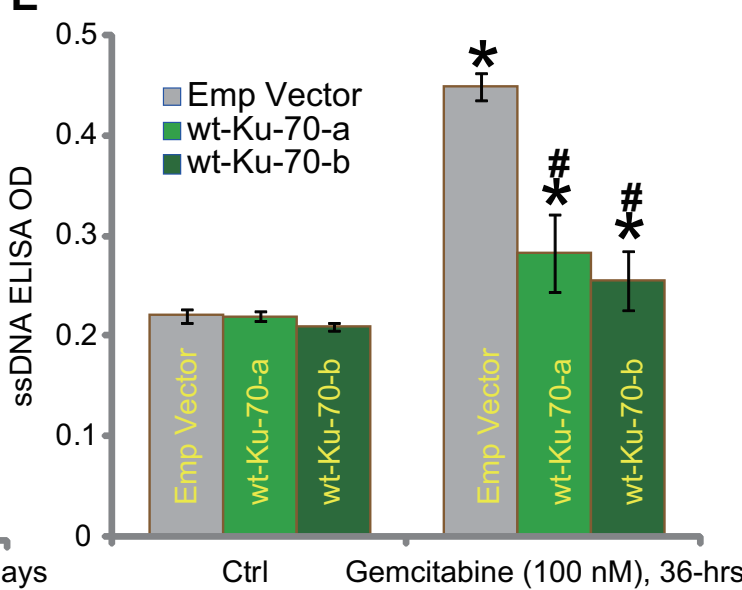


Figure 4

A.

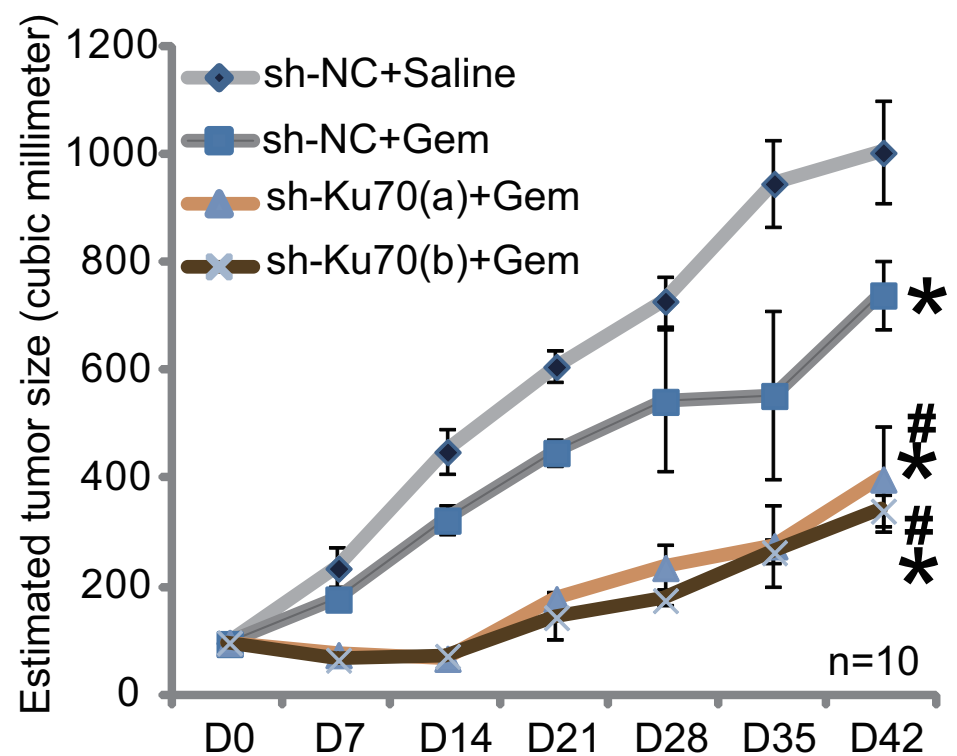

B.

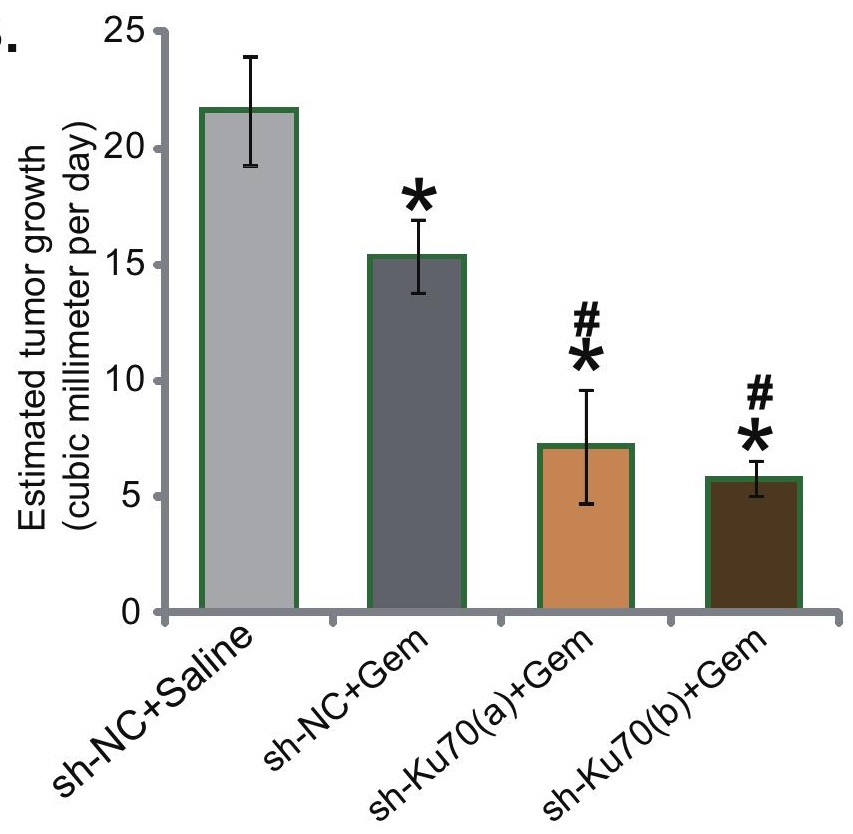

C.

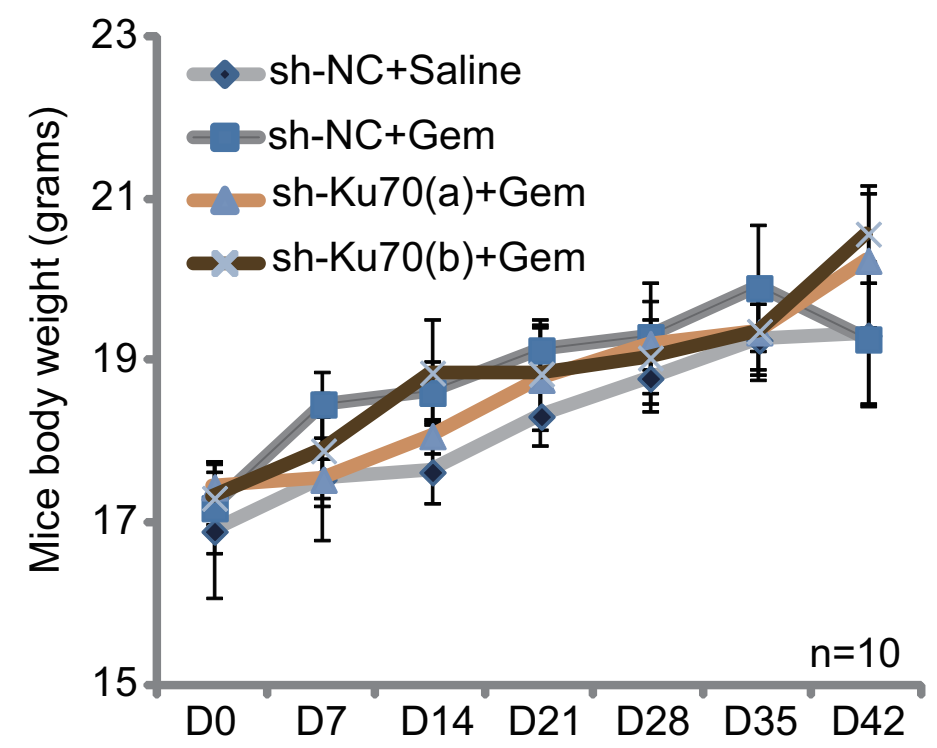

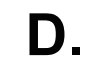

Tumor lysates (at Day-7) Tumor lysates (at Day-14)

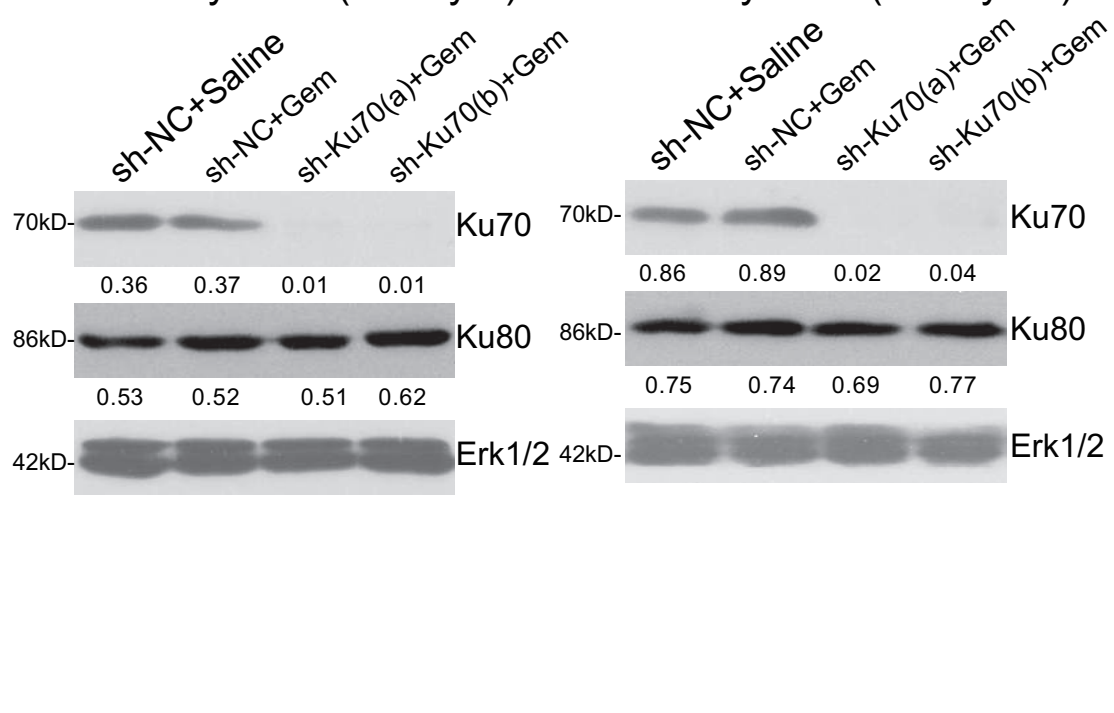

